\title{
Operational Noise Assessment in an Open Cycle Power Plant: Study on the Existing Acoustical Performance.
}

\author{
Helmi A Halim ${ }^{1 *}$, Gasim Hayder ${ }^{2}$ \\ ${ }^{1}$ Reliability \& Planning Department, Malakoff Power Berhad, Bt 2, Jalan Seremban, 71000 Port Dickson, Malaysia \\ ${ }^{2}$ Sustainable Technology and Environmental Research Group, Institute of Energy Infrastructure, Department of Civil Engineering, \\ Universiti Tenaga Nasional, Jalan IKRAM-UNITEN, 43000 Kajang, Selangor, Malaysia \\ *Corresponding author E-mail: helmi.halim@malakoff.com.my
}

\begin{abstract}
This research is an acoustical performance study in an open cycle peaking gas turbine power plant located at the southern coast of Peninsula Malaysia. Referring to its location at the middle of residence and tourism area, throughout its operation years, several complains from public has been lodged about the excessive operational noise at the perimeter of the plant and statistically from the last decade, twenty-four (24) noise related public complains were recorded. This issue has initiated a study to determine the current operational noise level as well as the effectiveness of the existing noise barriers. Based on EIA approval, the boundary noise for the operation should be limited to $55 \mathrm{~dB}(\mathrm{~A})$ at any time. The field measurement of noise level at two (2) different locations are assessed in-situ and continuously noise monitoring covering all the plant's operational regime and at different plant operational pattern. These results are compared with the initial noise report during its early year of operation in 1999. Finally the hypotheses then compared with the referenced legislations. Results from all the methodologies show the current operational noise level of the plant are within the permissible limit, however the overall operational noise of the plant is increasing compared with 1999 report due to the increasing of surrounding activities as well as deteriorating of current engineering and natural noise barrier arrangement. Mitigation plans has been recommended to the management to minimize the impact of the excessive noise to surrounding residence and to the plant workers. On top of that, the theoretical and commercial implication of the research is also discussed.
\end{abstract}

Keywords: Acoustical Performance; Noise Barrier; Operational Noise; Public Complaint.

\section{Introduction}

This research is initiated to study the current operational noise level as well as the effectiveness of the existing noise barriers at the open cycle power plant located at the southern coast of Peninsula Malaysia. The dual fueled power plant consists of 4 unit of gas turbines and in its $23^{\text {rd }}$ year of operation.

The open cycle gas turbine power plant is located at the southern coast of Peninsula Malaysia, sits inside a perimeter of $2.2 \mathrm{~km}$. The plant design is surrounded by 55 meter buffer zone which consist of planted and natural forestry which acts as natural noise absorption. This secondary forestry is also act as green belt (GB) to absorb and dampened the operational noise.

The plant is surrounded by the residential areas consist of 3 main residential area at the north and west side of the plant. The residential areas give a population of approximately 700 residences. Besides that, the commercial area consist of shop houses and small industrial area covers majority of the east side of the plant. The plant is also located near the tourism area which has 3 popular beach resorts built side by side. The resorts are becoming one of the most popular tourist attraction which attracts more than 50,000 tourists local and international yearly.

With the location surrounded by the public interest area, it is very important for the plant to comply with the Environmental Impact Assessment (EIA) and Department of Environment (DOE) regulation on the operational noise. The noise issues is being a sensitive issue since the residences had launched a protest on the plant's operational noise since the beginning of the plant's operation. Therefore, this issue is given the first priority by the management of the plant to ensure its conformation towards the regulation.

Since the proposal of the plant's EIA in 1994, the issue of noise is being the main concern. Taking public interest into their main priority, the EIA suggested a stringent mitigation plans to minimize the impact of excessive noise to the public.

During its operation years, several complaints from public regarding the excessive noise had being launched through such as Department of Safety \& Health (DOSH), DOE, Local Government Authorities, Biro Pengaduan Awam and etc. Based on the record from the plant management, during the previous decade of the operation (2007 - 2017), at least 1 complain being recorded per year and it accumulated a total of 24 complains received from the residences within the period.

Although most of the claims were false claims, the plant management should be more proactive in conforming the environmental noise requirements by the authorities and by the EIA 1994 especially during the operation of the plant [1].

On top of that, EIA also recommended a noise level measurement at the plant perimeter which complied with all the recommendation and guidelines incorporated to the operation of the plant especially on the level of noise exposure as highlighted by Factories \& Machinery Act (Noise Exposure) Regulation 1989 [2].

Previously, a study was conducted to determine the noise level of the plant's operation in 1999 [1] after the commissioning of the plant by Institute of Noise \& Vibration, Universiti Teknologi Ma- 
laysia (UTM) on behalf of DOE Malaysia. This study is to obtain the baseline of the operational noise level after 23 years of operation.

This research is initiated to determine its significant to the current noise level of the plant's operation and to gauge the potential noise exposure to the plant worker as per Factories \& Machinery Act (Noise Exposure) Regulation 1989 as well as the effectiveness of current noise barriers towards the recommendation and required level spelled out in the EIA in 1994 and also to the current legislation in Malaysia.

This research as well acted as a basis to the noise level permitted by the operation of the plant which being subjected to the public complains throughout its operating years.

\section{Methodology}

\subsection{Location of Assessment Points}

For this research, the location of measurement points is determined by the distance from the source of noise and the public which is the residential area as in Figure 1. The location of existing noise barriers is also taken into consideration in choosing the measurement points as the effectiveness of the barrier is also being derived from the result.

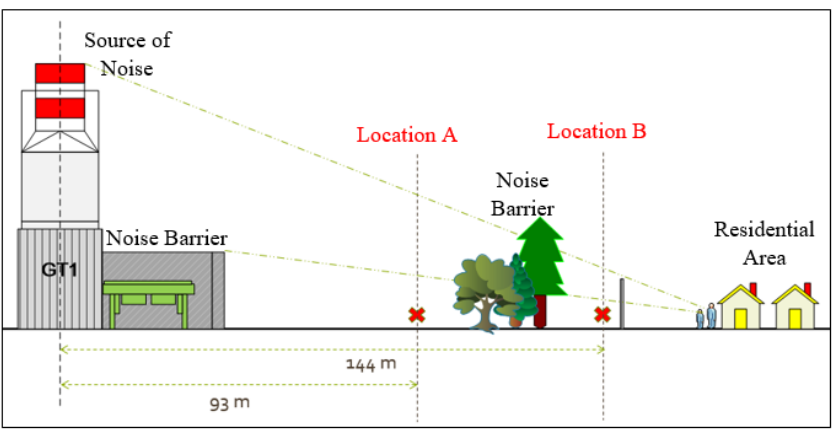

Fig. 1: Schematic Diagram of the Measurement Points

\subsection{In-Situ Measurement}

For this research, the device used is the station owned RION NA27 one third octave real time analyser with low frequency module (from $11 \mathrm{~Hz}$ to $2000 \mathrm{~Hz}$ frequency span) fitted with RION NH-17 Pre-Amplifier and RION UC-52 microphone $12 \mathrm{~mm}$ precision microphone.

The on-site measurement at the steady state condition is taken on various operational patterns and load variation at the designated locations using linear and A-weighted spectrum.

\subsection{Continuous Noise Monitoring}

The monitoring of continuous boundary noises was performed by using a pre-calibrated integrated high precision sound level meter. $\mathrm{LA}_{\mathrm{eq}}, \mathrm{LA}_{10}$ and $\mathrm{LA}_{90}$ were recorded at 15 minutes interval for each sampling locations in a period of 24 hours. The location of sampling is the same as the assessment location as on-site measurement.

The measurement is done using Sound Level Meter: Sper Scientific Model 850013 Advanced Data logging Sound Meter [3].

The " $A$ " weighting network had been used for sound pressure level measurements for equivalent $\mathrm{LA}_{\mathrm{eq}}$ and statistical centile $\left(\mathrm{LA}_{10}\right.$ and $\left.\mathrm{LA}_{90}\right)$ readings.

\subsection{Plant Operational Pattern}

In order to do the comprehensive study, the plant running pattern should be identified and analysed which combination will give the most significant impact to its operational noise emission. For an open cycle cyclic plant, the running pattern of the unit is based on the load demand. The Malaysian Grid is managed by NLDC and the plant is subjected to demand needs during peaking hours.

For this purpose, the proposed test condition and load distribution for the measurement is established based Table 1 and Table 2

Table 1: Proposed Test Condition

\begin{tabular}{|c|c|}
\hline Gas Turbine & Condition \\
\hline GT1 & Single GT Operation \\
\hline GT2 & Single GT Operation \\
\hline GT3 & Single GT Operation \\
\hline GT4 & Single GT Operation \\
\hline GT1 and GT2 (BLOCK 1) & Combination Operation \\
\hline GT3 and GT4 (BLOCK 2) & Combination Operation \\
\hline GT1, GT2, GT3 and GT4 & All unit Operation \\
\hline
\end{tabular}

Table 2: Proposed Load Distribution

\begin{tabular}{|c|c|}
\hline Load Distribution & Output \\
\hline Base load (maximum load) & $110 \mathrm{MW}$ \\
\hline Part load & $50 \mathrm{MW}$ \\
\hline Full speed no load (zero load) & $0 \mathrm{MW}$ \\
\hline
\end{tabular}

For this research purposes, in order to obtain the optimum reading of the operational noise of the plant, the measurement is taken during all unit in operation. This is the maximum operational noise emitted by the plant during its routine operation and anticipated to be the most significant operation pattern to this kind of power plant.

\section{Result}

\subsection{Continuous Noise Monitoring Summary}

The continuous monitoring is done for 24-hours continuous period where the data is taken in 15 minutes sampling time at both test locations [1]. The setup of the measurement equipment is shown in Figure 2.

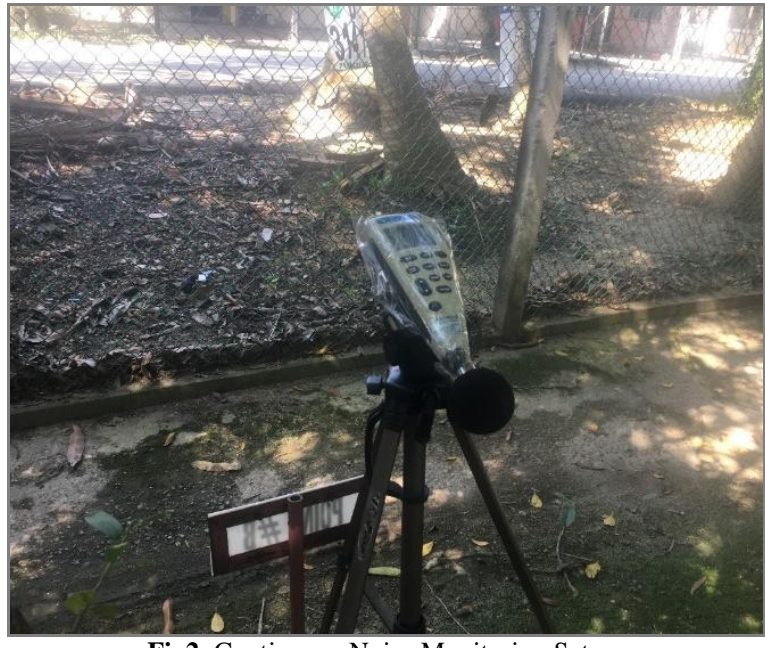

Fig2: Continuous Noise Monitoring Set up

Summary of the monitoring for the boundary noise measurements in term of exceeding permissible sound levels $\left(\mathrm{LA}_{\mathrm{eq}}\right)$ at both locations is presented in Table 3 .

Table 3: Continuous Noise Monitoring Summary

\begin{tabular}{|c|c|c|}
\hline Test location & $\begin{array}{c}\text { Day-time average } \\
\text { reading }\end{array}$ & $\begin{array}{c}\text { Night-time average } \\
\text { reading }\end{array}$ \\
\hline $\mathrm{A}$ & $50.1 \mathrm{dBA}$ & $47.6 \mathrm{dBA}$ \\
\hline $\mathrm{B}$ & $50.0 \mathrm{dBA}$ & $49.8 \mathrm{dBA}$ \\
\hline
\end{tabular}

Based on the analytical data from the monitoring for daytime period, Point A and Point B recorded no noise reading exceeded the 
limit in however, $8.3 \%$ and $2.8 \%$ of the readings exceeded the DOE's night-time permissible limit for point A and B respectively. The overall results of boundary noise monitoring from the two (2) sampling points show that the average of LAeq measured at Point $A$ and Point $B$ were well within and below the recommended level of $65.0 \mathrm{dBA}$ at daytime and $55.0 \mathrm{dBA}$ at night-time, respectively.

\subsection{On-site/ In-situ Measurement}

The data is taken during the schedule period at both locations of measurement during day time and night time in 15 second sampling time according to the test condition and load distribution [1], as shown in Figure 3.

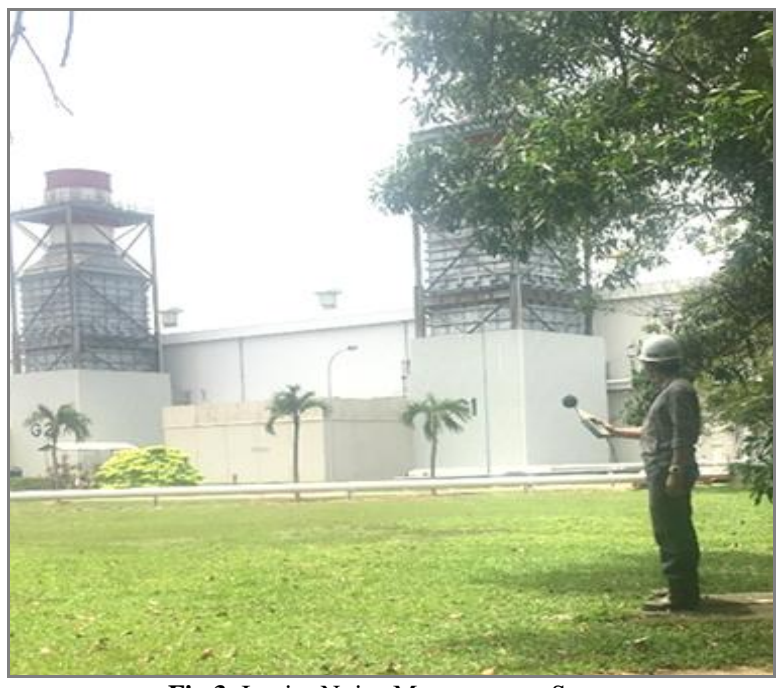

Fig 3: In-situ Noise Measurement Setup

For comparison purposes, the median value is taken from the measurement data as in Table 4.

Table 4: On-Site Measurement Median Data

\begin{tabular}{|c|c|c|}
\hline & $\begin{array}{c}\text { Night-time } \\
\text { Measurement (dBA) }\end{array}$ & $\begin{array}{c}\text { Day-time } \\
\text { Measurement (dBA) }\end{array}$ \\
\hline A & 61.5 & 62.6 \\
\hline B & 54.9 & 54.7 \\
\hline
\end{tabular}

\section{Result Hypothesis}

\subsection{Overall Reading}

The compilation for comparison of all the relevant data is illustrated in the following Figure 4.

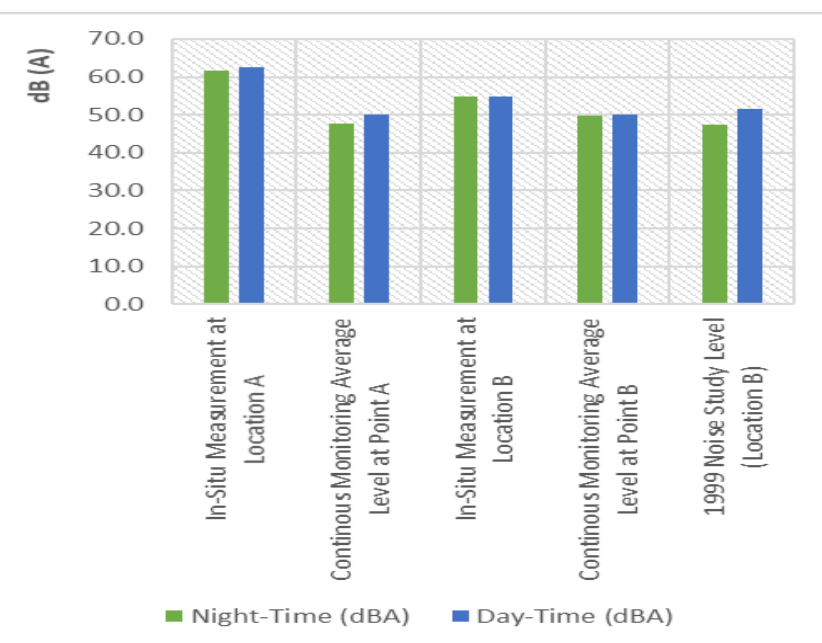

Fig. 4: Noise Measurement Result from All Methodologies
Base on the data taken at both locations, it is clearly indicates that the noise measurement during day time is slightly higher than the night time measurement although at the same load distribution. This is mainly due to the surrounding noise which add up to the measurement such as noise from moving vehicles at surrounding, bird chirping, air craft pass-by, human activities and etc. These outdoor environment is the influential factor for the increase of the day time noise [11].

The noise sampling taken in 1999 is lower than the noise sampling taken in this research in 2017. This discrepancy is maybe due to the different noise measurement methodology and also using a different noise measuring meter. In 1999, the noise reading was taken using RION SA-27 [1]. This might have some discrepancy in terms of the sensitivity and also outdoor activity during that particular time. The surrounding area is developing fast from 1995 to 2017 and the intensity of outdoor activities is also plays important role for this variance.

Overall, data measurement taken is still under the permissible limit set in the Environmental Impact Assessment in 1994 [7] which is $55 \mathrm{~dB}(\mathrm{~A})$.

Comparing the noise measurement at Point An and $\mathrm{B}$, it can be concluded the reduction of noise level taken on both locations is due to the effectiveness of the existing noise barrier which is generally the green belting around the parameter of the plant. The green belt is acted as natural absorption which dampened the noise density as well as low frequency vibration coming from plant operation.

\subsection{Noise Exposure}

The Factories \& Machinery Act (Noise Exposure) Regulation 1989 highlighted that no employees of the plant should be exposed to the limit $90 \mathrm{~dB}(\mathrm{~A})$ and above without any personal protection [7].

Based on the measurement results, the current noise level at the plant perimeter during operation, ranging between $49.0 \mathrm{~dB}$ (A) to $62.6 \mathrm{~dB}(\mathrm{~A})$, is way below the maximum exposure limit at any time of a day. This means the employees of the power plants are not to abide by the First Schedule of this regulation and can proceed with their activities without wearing any hearing protection.

\section{Conclusion \& Recommendation}

\subsection{Conclusion}

From the analysis, it can be concluded that the existing noise barriers within the plant's perimeter are sufficient to control and to mitigate the excessive operational noise from the power plant.

The current noise level at the selected location has been measured based on several methodologies. All the results show that the current noise level is within the allowable limit specified in the EIA, the report by Department of Environmental Malaysia in 1999 and the noise exposure limit as highlighted in by Factories \& Machinery Act (Noise Exposure) Regulation 1989. On top of that, current noise level during in 2017 is $6.21 \%$ higher in daytime and $16.07 \%$ higher during night-time compared with the study in 1999.

Clearly the noise level is within the regulatory limit, however, the noise measurement is evidently increasing compared with the level taken in 1999. This may be referred to the ageing factor on most of the equipment which emitted high pitch noise and vibration.

Plant management should continue their approach to maintain the noise barrier in terms of noise insulation and noise absorption in order to control the excessive operating noise from polluting the environment.

From the reading at 2 measurement locations (namely Location A and Location B), Location A is located before the case concerned noise barrier and Location $\mathrm{B}$ is located after the noise barrier which is the green belting along the perimeter of the plant. The 
result indicates that there is a reduction of noise level from Location A to Location B by $12.62 \%$ in day time and $10.73 \%$ in night time. This is the initial indication of the current plant's noise barrier is effective to reduce or control the boundary noise level of the plant during its operation.

\subsection{Recommendation for Improvement}

From the analysis, some recommendation can be made in order to improve the situation as highlighted in the research objectives. Generally the current noise barrier is efficient, however, several improvement can be conducted to enhance the effectiveness and as well as to minimize the impact of excessive noise from plant operational noise.

The overall operational noise is still under allowable limit, however, there is some spike on the noise level due to ageing acoustic absorption. Generally, the turbine hall wall itself act as the acoustic absorption. The wall surface will be filled by mesh galvanized wire sheet filled the fibres called 'Rockwool' to absorb the noise wave emitted by the operation of the turbines [8]. Due to the ageing factor, the Rockwool is thinning. This will allow the noise to leak out to environment. The Rockwool thickness needs to be repaired or replenish to control the sound emission from going out from the GT hall.

The plant outline configuration is already has a $55 \mathrm{~m}$ distance from the residence area which acted as a buffer zone and natural acoustic absorption as required in the EIA [1]. It is recommended to densify the barrier by planting more trees and bushes in order to control the noise [9].

The noise limit is maintained at maximum of $70 \mathrm{dBA}$ inside the plant and maximum of $60 \mathrm{dBA}$ at the perimeter fence of the plant indicating the effectiveness of noise barrier [10], and reduced impact to the nearby residences which just approximately 10 meter away from the boundary of the plant.

\section{Acknowledgement}

Appreciation is acknowledged to the management of the Power Plant which the research is executed for all the permissions and logistics in order to complete this research.

\section{References}

[1] Universiti Kebangsaan Malaysia (1999) Noise \& Vibration Study Report. Department of Environmental Malaysia

[2] Halim, H., Abdullah, R., Ali, A. A. A., \& Nor, M. J. M. (2015). Effectiveness of existing noise barriers: compari-son between vegetation, concrete hollow block, and panel concrete. Procedia Environmental Sciences, 30, 217-221.

[3] Environmental Noise Boundary Monitoring Report, October 2017 Airwastewater Management Sdn. Bhd.

[4] D’Hondt, E., Stevens, M., \& Jacobs, A. (2013). Participatory noise mapping works! An evaluation of participatory sensing as an alternative to standard techniques for environmental monitoring. Pervasive and Mobile Computing, 9(5), 681-694.

[5] Brittain, F. (2004). Noise modeling using ISO 9613-2 for designing facilities to meet a not-to-exceed community noise limit. In NoiseCon 04. The 2004 National Conference on Noise Control Engineering Institute of Noise Control Engineering Transportation Research Board.

[6] Ismail, A. R., Nor, M. J., Mansor, M. R., Tahir, M. F., \& Zulkifli, R. (2009). Environmental noise assessment and modeling in Malaysia: A comparative monitoring study. European Journal of Scientific Research, 30(2), 236-244.

[7] Mohtar, M. (1999). Factories and machinery (Noise exposure) regulations 1989 (No. L-0537). MOPGC.

[8] Mokhtar, Mohzani \& Kamaruddin, Sahrul \& Khan, Zahid \& Mallick, Zulquernain. (2007). A Study on the Effects of Noise on Industrial Workers in Malaysia. Jurnal Teknologi. 46. 17-30.

[9] Rao, P. S., Gavane, A. G., Ankam, S. S., Ansari, M. F., Pandit, V. I., $\&$ Nema, P. (2004). Performance evaluation of a green belt in a pe- troleum refinery: a case study. Ecological Engineering, 23(2), 7784.

[10] Kisku, G. C., \& Bhargava, S. K. (2006). Assessment of noise level of a medium scale thermal power plant. Indian Journal of Occupational and Environmental Medicine, 10(3), 133.

[11] Jiang, L., \& Kang, J. (2017). Perceived integrated impact of visual intrusion and noise of motorways: Influential factors and impact indicators. Transportation Research Part D: Transport and Environment, 57, 217-223. 